2 Taves DR. Determination of submicromolar concentrations of fluoride in biological samples. Talanta 1968;15:1015-23.

${ }^{3}$ Ekstrand J, Alván G, Boréus I.O, Norlin A. Pharmacokinetics of fluoride in man after single and multiple oral doses. Eur $\mathbf{f}$ Clin Pharmacol 1977;12:311-7.

${ }^{4} \mathrm{Kwit}$ NT, Hatcher RA. Excretion of drugs in milk. Am $\mathcal{f}$ Dis Child 1935;49:900-40.

${ }^{5}$ Holland RI, Hongslo JK. Fluoride, fluoride resistance and glycolysis in cultured cells. Acta Pharmacol Toxicol 1978;43:240-5.

(Accepted 19 fune 1981)

Karolinska Institutet, School of Dentistry, Box 4064, S-101 04 Huddinge, Sweden

J EKSTRAND, ODONT D, assistant professor, department of cardiology

Karolinska Hospital, S-104 01 Stockholm, Sweden

L O BOREUS, MD, head, department of clinical pharmacology

P DE CHATEAU, MD, assistant professor, department of paediatrics

\section{Fatal subarachnoid haemorrhage in young women: role of oral contraceptives}

After a report was published in 1977 from the Royal College of General Practitioners' Oral Contraceptive Study, ${ }^{1}$ which suggested a possible association between use of oral contraceptives and haemorrhagic stroke, a case-control study of fatal subarachnoid haemorrhage was carried out by Inman. ${ }^{2}$ This showed a small, but not statistically significant, increased risk of subarachnoid haemorrhage in association with oral-contraceptive use in the absence of hypertension, though it was considered that use of oral contraceptives might be related indirectly by raising blood pressure. We report the results of a further study of this problem.

\section{Methods and results}

The methodology is discussed in detail elsewhere. ${ }^{3}$ We obtained death certificates for 446 women aged 15-44 years certified as dying of subarachnoid haemorrhage in England and Wales during 1978. Two hundred and seven cases were excluded; because there was insufficient evidence for the diagnosis (162), because the records could not be traced (32), or because death occurred after operation or during pregnancy and puerperium (13). Information about the remaining women and about two controls, selected at random ${ }^{3}$ for each woman and matched for sex, age, and marital status, was sought from their general practitioners. A further 71 deaths were not investigated fully because the general practitioner could not be traced or felt unable to help. Our results are therefore based on the 168 completed case-control sets.

Thirty-six (21\%) cases were aged 15-29 years, 73 (43\%) aged 30-39 years, and $59(35 \%)$ aged $40-44$ years. The controls were matched for age and marital status and had a similar social-class distribution. In $95(57 \%)$ cases the diagnosis was based on post-mortem findings, in $8(5 \%)$ on operative findings, in $13(8 \%)$ on findings on diagnostic angiography or computed tomography scan and in $52(31 \%)$ on clinical findings only (results of lumbar puncture or the presence of subhyaloid haemorrhage). The table shows that there were no significant differences between cases and controls in their current or past use of oral contraceptives. Similar results were obtained when cases with only clinical evidence of subarachnoid haemorrhage were excluded and when separate age groups were analysed.

The known risk factors for subarachnoid haemorrhage were more prevalent in cases than controls: hypertension ( 33 cases $(20 \%)$, 9 controls $(3 \%)$ ), a history of pre-eclamptic toxaemia $(11(7 \%)$ and $6(2 \%))$, renal disease $(5$ $(3 \%)$ and $2(1 \%))$, and a previous subarachnoid haemorrhage $(9(5 \%)$ and none). Oral-contraceptive use in women with and without risk factors is shown in the table. The 33 cases diagnosed as hypertensive showed a higher rate of past use $(55 \%)$ and a lower rate of current use $(9 \%)$ when compared with non-hypertensive cases. Twelve of the 18 past users had been diagnosed as hypertensive when using oral contraceptives and had stopped using the pill for this reason. Data on smoking were available for only $75(45 \%)$ cases and $95(28 \%)$ controls, but among these women there were more smokers in cases $(61 \%)$ than controls $(32 \%)$. Although fewer cases who were using oral contraceptives were taking a preparation containing less than $50 \mu \mathrm{g}$ oestrogen ( 9 out of 27 compared with 36 out of 65 ) the difference was not significant.

\section{Comment}

This study confirms the importance of known risk factors for subarachnoid haemorrhage. There have been five previous studies ${ }^{2} 45$ examining the role of oral contraceptives in the aetiology of subarachnoid haemorrhage. Three showed a statistically significant increase in risk associated with oral-contraceptive use, ${ }^{24}$ one a small but not significant increase in risk, ${ }^{2}$ and one no association. ${ }^{5}$ The present study shows a small but not significant increase in risk. As Inman has suggested ${ }^{2}$ oral contraceptives might act indirectly by raising blood pressure: 15 of the 33 patients with hypertension were first found to have raised levels of blood pressure while taking the pill. This effect was not great enough, however, to result in a significant difference in oral-contraceptive use. These findings taken with those of earlier studies suggest that both the relative and absolute risks of subarachnoid haemorrhage associated with use of oral contraceptives are small and are probably associated with the hypertensive effect of the pill.

We would like to thank the many people who have helped with this study and to acknowledge the financial support of the Medical Research Council.

1 Royal College of General Practitioners. Mortality among oral-contraceptive users. Lancet 1977; ;i :727-31.

2 Inman WHW. Oral contraceptives and fatal subarachnoid haemorrhage. Br Med F 1979;ii:1468-70.

3 Adam SA, Thorogood M, Mann JI. The pill and myocardial infarction revisited: the effects of new preparations and prescribing patterns. Br $\mathcal{F}$ Obstet Gynaecol (in press).

${ }^{4}$ Royal College of General Practitioners. Further analyses of mortality in oral contraceptive users. Lancet 1981 ; : 541-6.

${ }^{5}$ Vessey MP, McPherson KC, Yeates D. Mortality in oral contraceptive users. Lancet 1981 ; i:549.

(Accepted 12 fune 1981)

Department of Community Medicine and General Practice, Oxford OX1 3QN

M THOROGOOD, BSC, research assistant

S A ADAM, MB, MRCP, MRC research fellow (present appointment: senior registrar in community medicine, Oxford Regional Health Authority)

J I MANN, DM, PHD, university lecturer

Risk factors of cases and controls by use of oral contraception. Results are numbers (\%)

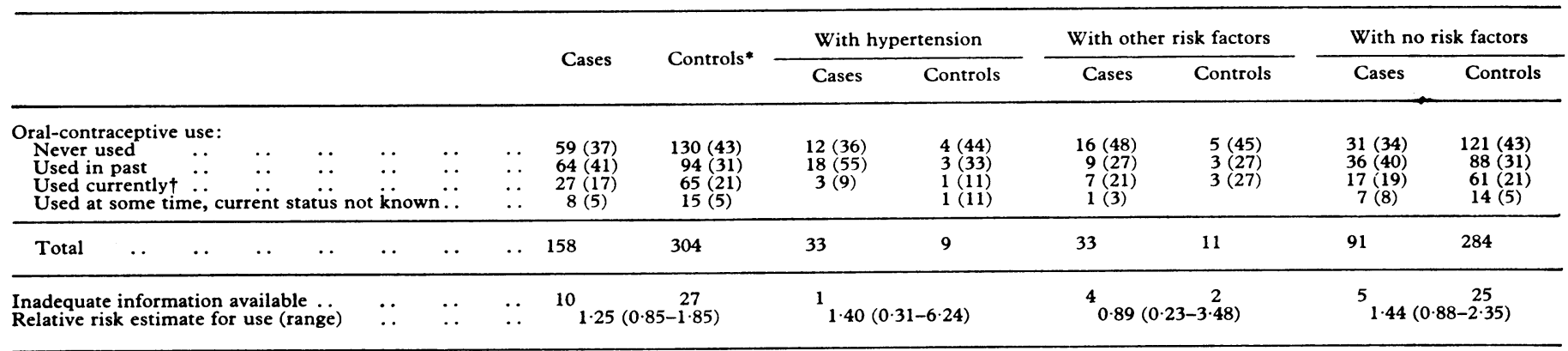

* In five instances data on only one control were available.

"Currently = At the time of death for cases, and at the time of death of the relevant case for each control. 